\title{
Efficacy of Ultrasonography Guided PRP on Chronic Insertional Tendinitis of Achilles tendon
}

\author{
Abdul Haque ${ }^{1 *}$, Sheik Md. Abdullah Rafi ${ }^{2}$ \\ ${ }^{1}$ Associate Professor, Department of Orthopedic Surgery, Cumilla Medical College, Cumilla, Bangladesh \\ ${ }^{2}$ Consultant, Cumilla Trauma Center, Cumilla, Bangldesh
}

DOI: $10.36347 /$ sjams.2020.v08i09.005

| Received: 30.08 .2020 | Accepted: 07.09.2020 | Published: 09.09.2020

*Corresponding author: Dr. Abdul Haque

Abstract

Original Research Article

Background: Chronic insertional tendinitis of Achilles tendon is a common condition that can be challenging to treat. There are many modalities used as treatment ranging from physiotherapy, injections, shockwave therapy to surgical intervention. Platelet Rich Plasma (PRP) has increased in popularity recently as a treatment option for Achilles Tendinopathy. It contains growth factors that might accelerate healing and speed up the recovery of this condition. Aim of the study: The aim of this study was to evaluate the efficacy of ultrasonography guided PRP on chronic insertional tendinitis of Achilles tendon. Methods: This prospective observational study was conducted in Cumilla Trauma Center, Cumilla, Bangladesh during the period from January 2018 to July 2019. In total 52 patients with chronic insertional tendinitis of Achilles tendon selected and completed the full tenure of ultrasonography guided PRP treatment procedure were finalized as the study people. The study was approved by the ethical committee of the mentioned institute. Proper written consents were taken from all the participants. All the data were collected through a pre designed questioner and the findings were displayed in MS Excel tables and necessary figures. Result: In this study, the pre-test the mean VAS (Visual Analogic Score) of the participants was 7.6. After 10 weeks of applying PRP it was found 1.8 and after 24 weeks it was found 1.3. So this treatment method ensures very satisfactory mean VAS scores of the participants. In this study when we analyzed the levels of patient's satisfaction we found, 59.62\% $(n=31)$ participants were satisfied whereas $36.54 \%(n=19)$ were moderately satisfied. Besides these, in this study we found only 2 participants who were not satisfied at all. Conclusion: Platelet-rich plasma US-guided injection is a safe procedure with no complications. Ultrasound is helpful to manage the follow-up, demonstrating the remodeling of the tendinosis area in correlation to the healing process and the clinical improvement.

Keywords: Ultrasonography, Platelet Rich Plasma (PRP), Tendinitis, Achilles tendon.

Copyright @ 2020: This is an open-access article distributed under the terms of the Creative Commons Attribution license which permits unrestricted use, distribution, and reproduction in any medium for non-commercial use (NonCommercial, or CC-BY-NC) provided the original author and source are credited.

\section{INTRODUCTION}

Achilles tendinopathy is a common condition affecting the Achilles tendon, characterized by pain, swelling, weakness and stiffness [1]. Its etiology is likely to be multifactorial with repetitive exposure to trauma leading to tendon degeneration, micro-tears and impairment of tendon repair [2]. Although previously thought to be an inflammatory condition, clinical, imaging and histological examinations have shown that the process is predominantly degenerative with local disruption of the collagen matrix and micro-tears [3]. It is characterized by hyper-cellularity, fibroblast proliferation, and collagen disorganization [4] more commonly termed angio-fibroblastic hyperplasia [5]. There is no agreement on the ideal treatment modality for Achilles tendinopathy and this remains a complex difficult challenge [6]. This has led to the introduction of a large number of conservative treatments including rest, immobilization, analgesia, NSAIDs, orthotics, physiotherapy, local injections, extracorporeal shock wave therapy and topical glyceryl trinitrate [5]. In an 8year follow-up study, $29 \%$ failed conservative treatment [7] and were surgically treated. Surgical treatments options that have been explored include operative debridement, autogenous flexor halluces longus or flexor digitorum longus tendon transfer, and allograft augmentation [6]. Platelets play an active role in homeostasis during the initial inflammatory phases. Platelets become activated through the adhesion process, aggregation, clot formation, and release a range of mitogenic and strong inflammatory mediators into the local microenvironment [8]. Dense granules, alphagranules and lysosomes release proteins, growth factors (GF), and chemokines and coagulation factors. These substances act in a concerted and finely regulated 
manner to influence and promote tissue repair functions [9]. GF released from alpha granules are summarized in table 1[10]. There are a lot of study and data regarding the histological features of such diseases. Histological features in the early stages of the disease are focal areas of asymptomatic intra-tendineous damage without signs of inflammation [11]. Histological appearance of degenerative damage begins and progresses long before onset of symptoms. This delay causes late therapeutic approaches to be less successful [12]. Patellar tendinopathy, in which degeneration is located at the proximal enthesis, is also known as jumper's knee. This disease mostly affects young athletes and is associated with running, jumping, soccer, and volleyball [13]. Prevalence has been estimated to approx. $40 \%$ e $50 \%$ among professional volleyball players and $35 \% \mathrm{e} 40 \%$ among basketball players. Some intrinsic factors (e.g. gender, age and overweight) and extrinsic factors (e.g. functional overload and training errors) play an important role in the development of chronic tendinopathy [13]. Achilles tendinopathy is a common condition in the adult population. In $59 \%$ of patients it is related to sports activities, and $53 \%$ of them are runners [14]. At present, patients affected by jumper's knee and Achilles tendinopathy are primarily treated with rest, ice packs, physical therapy, non-steroidal anti-inflammatory drugs (NSAIDs) and steroid injections, although there is no evidence of the effectiveness of these therapies[14]. New theories of tendon regeneration have been focused on the role of platelets and the development of different therapeutic strategies in the treatment of chronic tendinopathy [15]. Platelets are involved in the mechanism of thrombus formation and participate in the healing process by removing necrotic tissues and stimulating regeneration and healing of the tissues [16]. Platelet-rich plasma (PRP) with a high concentration of platelets has been used in cardiac, maxillofacial, plastic, neuro, and orthopedic surgery [17]. More recently, PRP injections have been used to treat muscle, tendon, and cartilage injuries [18].

\section{OBJECTIVES}

\section{General Objective}

- To evaluate the efficacy of ultrasonography guided PRP on chronic insertional tendinitis of Achilles tendon.

\section{Specific Objective}

- To assess the gender and age distribution of the patients with chronic insertional tendinitis of Achilles tendon

\section{Methodology \& Materials}

This prospective observational study was conducted in Cumilla Trauma Center, Cumilla, Bangladesh during the period from January 2018 to July 2019. In total 52 patients with chronic insertional tendinitis of Achilles tendon selected and completed the full tenure of ultrasonography guided PRP treatment procedure were finalized as the study people. The study was approved by the ethical committee of the mentioned institute. Proper written consents were taken from all the participants. According to the inclusion criteria of this study presence of achilles tendinopathy with pain at palpation and during physical activity for at least three months and USG or magnetic resonance imaging (MRI) evidence of tendon degeneration and patients had previously non satisfactory outcome with other conservative treatments were included. On the other hand, according to the exclusion criteria of this study, systemic disease (Such as: diabetes, rheumatoid arthritis, coagulopathy, severe cardiovascular disease, infection and immune-suppression), intake of NSAIDs less than 28 days before the procedure were excluded. For clinical evaluation all the tasks were performed by the same Orthopaedic surgeon using the Visual Analogue Scores (VAS) scale which assessed pain and activity level. Clinical evaluation was performed before treatment-After PRP and needle fenestration procedure at 4, 10 and 24 weeks using the same criteria and at the 6 month follow-up evaluation, overall satisfaction with the treatment was asked using a semi-quantitative scale (poor, intermediate, good, excellent). We collected 20 $\mathrm{ml}$ blood for each procedure \& prepare about $3 \mathrm{ml}$ PRP using a korean kit named i-stem PRP kit which need two step cetrifugation method. After collecting the PRP we use a $18 \mathrm{G}$ needle for our injection procedure. Under direct sonoguidance, we inject PRP around the most thickened \& inflammed part of the tendon (evidenced by high vascularity \& echogenicity) \& retro-calcaneal bursa also (because many a time we found simultaneous RC bursitis with tendinitis). For ultrasound and Color Doppler evaluation all studies were performed by one single experienced musculoskeletal radiologist using high-resolution transducers and frequencies. For each procedure, US data and Color Doppler Flow Imaging data were recorded. All the data were collected through a pre designed questioner and the findings were displayed in MS Excel tables and necessary figures.

\section{RESULT}

The aim of this study was to evaluate the Efficacy of ultrasonography guided PRP on chronic insertional tendinitis of Achilles tendon. In this study after all the inclusion and exclusion procedures finally 52 subjects were finalized as the total study population. Among them 65\% $(n=34)$ were male whereas $35 \%$ $(n=18)$ were female. In analyzing the ages of the participants we found the highest 25 participants were from $41-60$ years' age group which was $48.08 \%$ of the total population. Then $30.77 \%(n=16)$ were from $21-40$ years' age group, $11.54 \%(\mathrm{n}=6)$ were from $\leq 20$ years' age group and the rest $09.62 \%(n=5)$ were from $>60$ years' age group. In this study, the pre-test the mean VAS (Visual Analogic Score) of the participants was 7.6. After 10 weeks of applying PRP it was found 1.8 and after 24 weeks it was found 1.3. So this treatment method ensures very satisfactory mean VAS scores of 
the participants. In this study when we analyzed the levels of patient's satisfaction we found, $59.62 \%(n=31)$ participants were satisfied whereas $36.54 \%(n=19)$ were moderately satisfied. Besides these, in this study we found only 2 participants who were not satisfied at all.

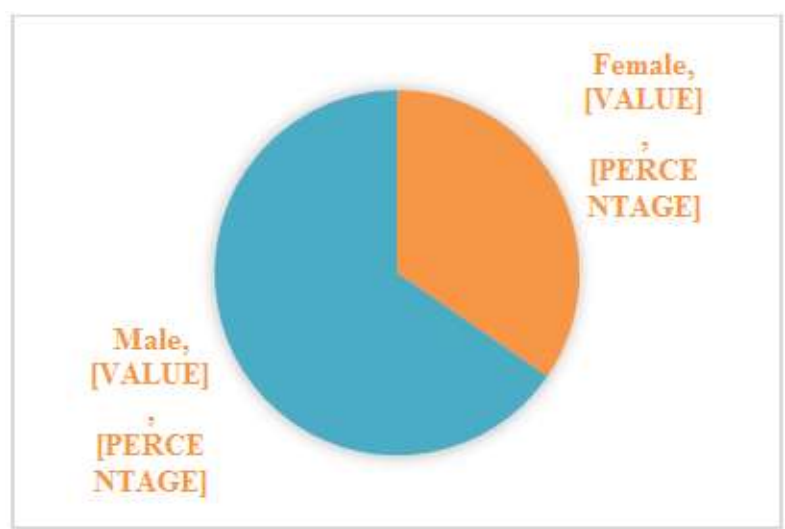

Fig-I: Age and gender distribution of participants $(\mathrm{N}=\mathbf{5 2})$

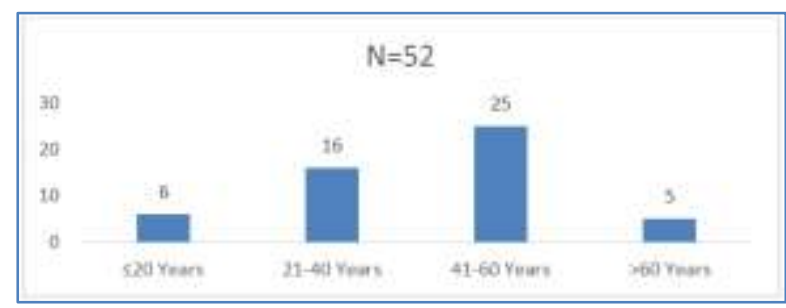

Fig-II: Age distribution of participants ( $\mathrm{N}=52)$

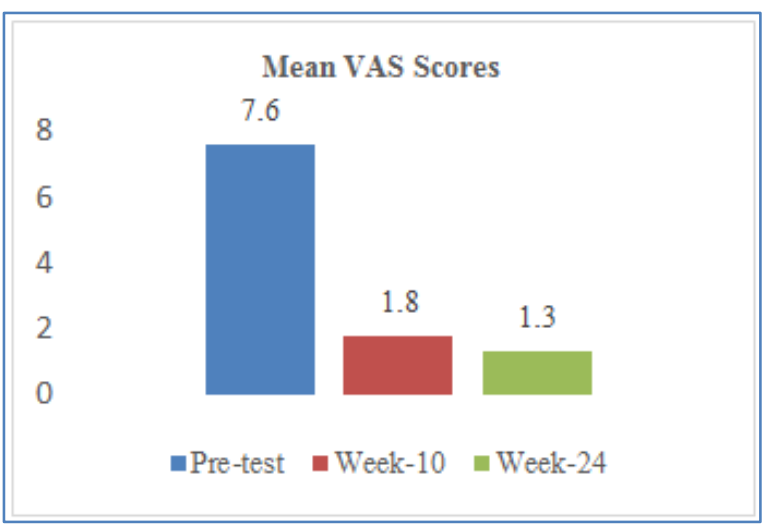

Fig-II: Mean VAS scores changes in participants $(\mathrm{N}=52)$

Table-I: Levels of satisfaction of participants $(\mathrm{N}=52)$

\begin{tabular}{|l|c|c|}
\hline \multicolumn{1}{|c|}{ Satisfaction level } & n & \% \\
\hline Satisfied & 31 & 59.62 \\
\hline Moderately Satisfied & 19 & 36.54 \\
\hline Not Satisfied & 2 & 3.85 \\
\hline
\end{tabular}

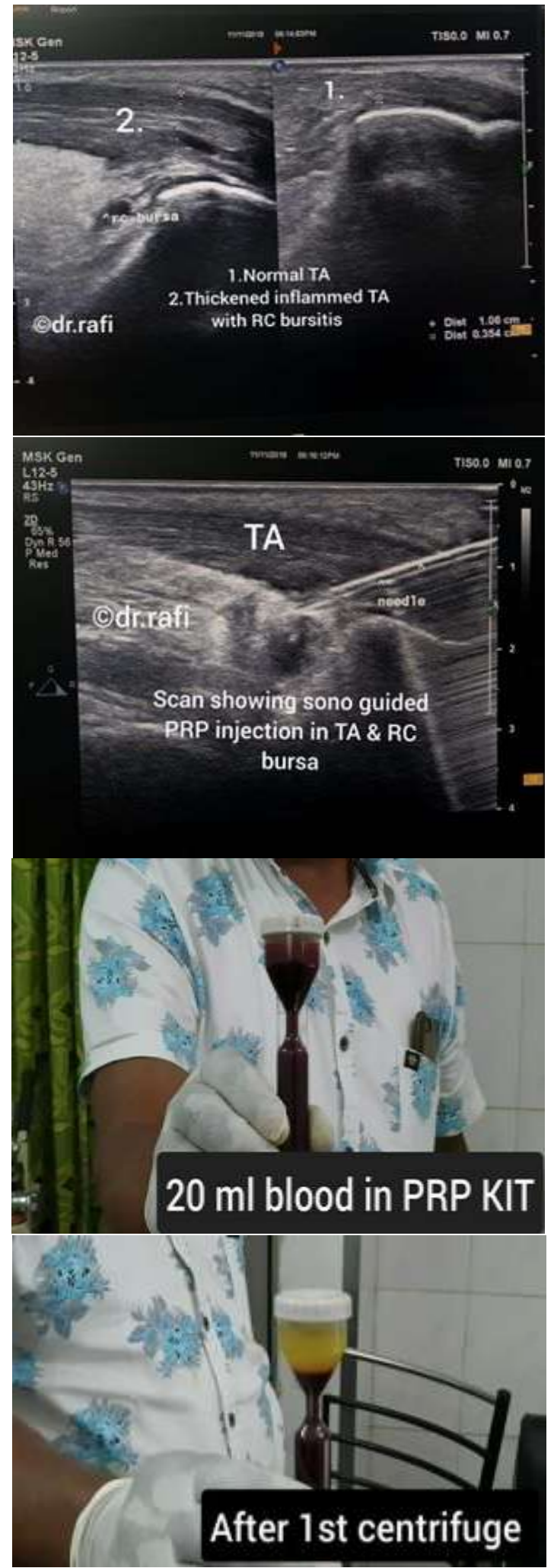




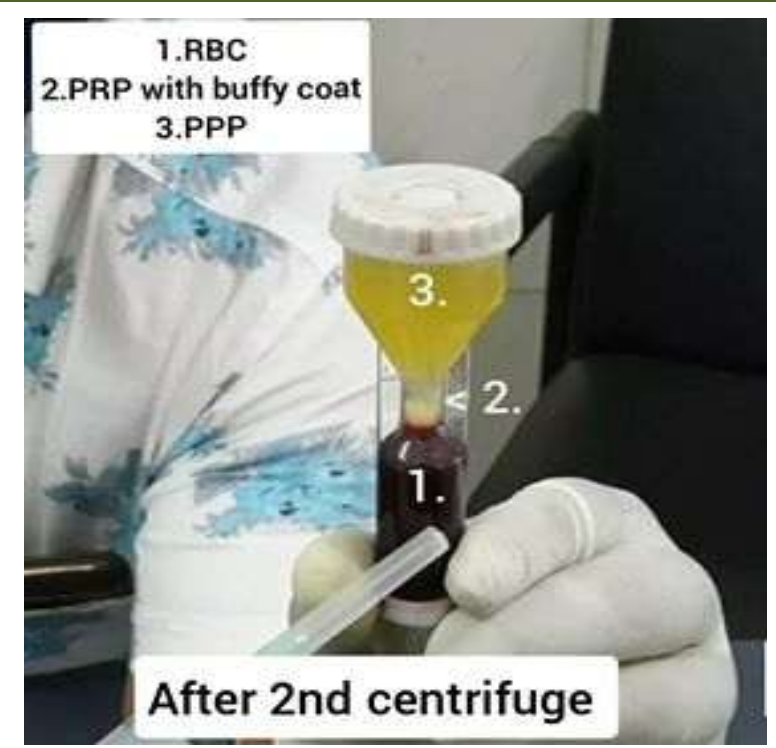

\section{DISCUSSION}

Basically, PRP was used first in oral and maxillofacial surgery but became more used for a wide variety of orthopedic procedures. The motto was to stimulate and promote healing processes by placing higher than normal concentrations of autologous platelets as well as growth factors at the site of tendon damage. In fact, the popularity of PRP have especially increased after receiving media attention for its use in professional athletes whose accelerated healing and return on the field or play-ground was credited to PRP treatment. Radiologists, especially those offering musculoskeletal ultrasound services in collaboration with sports medicine physicians as well as Orthopaedic surgeons significantly increased the number of USguided treatments for chronic tendinopathies, ligamentous or muscle injuries using PRP injections. Tendinosis occurs due to repetitive stress, overuse, degeneration, which lead to painful conditions affecting the quality of life, impairing mobility. Tendinitis is a inappropriate response occurring when breakdown of collagen fibers exceeds the rate of repair with collagen degeneration and fiber disorganization. There is hypercellularity, scattered vascular and neural ingrowth associated or not with para-tendinopathy where an inflammatory process can affect the synovial tendon sheath or adventitial para-tendon, but no histopathologic evidence of inflammatory cells. In fact, tendon healing is a slow process, and the resolution of symptoms precedes the end of healing process. Early return to full activity may lead to a progressive tendinosis. Our increasing knowledge, understanding and preliminary evidence from experimental data that GFs, present in plasma, boosts tendon repair mechanisms through recruitment, proliferation and differentiation of cells, has driven the use of PRP preparations in the treatment of Achilles tendinopathy [19]. However, the role and clinical effectiveness of PRP use in Achilles tendinopathy is at its infancy. Abate [20] outlines several theoretical and practical reasons about the possible cause of treatment failure. Animal studies were conducted on "surgically induced" tendons, so it was debatable whether these models mimic human pathology, where human tears usually occur in degenerated tendons. The pathway of chronic tendinopathies is very complex, involving many pathogenetic factors, including GFs, which operate at different stages of the disease [20]. Although all four studies [6] used leucocyte and PRP, different methods have been used to produce the PRP, which can result in a large variation in GF content, and in the kinetics of release [20]. The optimal PRP formulation is unknown, with platelet concentrations differing widely. The concentration of platelets in PRP can range from 2.5 to 8 times the concentration found in whole blood [20]. Moreover different formulations may be required at different stages of tendon healing or for patients with different histopathological or biochemical features [21]. The clinically applicable evidence on the role of PRP in Achilles tendinopathy to date stems mainly from the above studies. But due to their differing study protocols, comparison of results is difficult. These limitations are recognized by NICE [22], who acknowledge that "current evidence on the safety and efficacy of autologous blood injection for tendinopathy is inadequate in quantity and quality. Therefore this procedure should only be used with special arrangements for clinical governance, consent and audit or research". In our study, the pre-test the mean VAS (Visual Analogic Score) of the participants was 7.6. After 10 weeks of applying PRP it was found 1.8 and after 24 weeks it was found 1.3. So this treatment method ensures very satisfactory mean VAS scores of the participants. In this study when we analyzed the levels of patient's satisfaction we found, $59.62 \%(n=31)$ participants were satisfied whereas $36.54 \%(n=19)$ were moderately satisfied. Besides these, in this study we found only 2 participants who were not satisfied at all. There is a variation in the agreed gold standard of diagnosis, where the majority [6] used ultrasound and/or MRI in combination with physical examinations. An exception is de Vos et al. [23] who confirmed the diagnoses clinically. The classification of acute and chronic Achilles tendinopathy is unclear, with a variable mean duration of symptoms prior to enrolment in the studies. In the RCT, de Vos included symptomatic subjects for at least 2 months [23], whilst Owens included subjects with at least 6 months of symptoms [24]. Additionally, the degenerate tendon pathology seen in Achilles tendonopathy may not be responsive to PRP injections [20]. This makes it difficult to compare the studies and further research is needed.

\section{Limitations OF THE STUDY}

The sample was not randomized in our study. It was also a single centered observational study with limited sample. So, the findings of this study may not reflect the exact scenario of the whole country. 


\section{CONCLUSION AND}

\section{RECOMMENDATIONS}

Platelet-rich plasma US-guided injection is a safe procedure with no complications. Ultrasound is helpful to manage the follow-up, demonstrating the remodeling of the tendinosis area in correlation to the healing process and the clinical improvement. For getting more specific findings, we would like to recommend for conducting case-control studies regarding the same issue with larger sized sample.

\section{REFERENCES}

1. Khan KM, Maffulli N. Tendinopathy: an Achilles' heel for athletes and clinicians. Clinical Journal of Sport Medicine. 1998 Jul 1;8(3):151-4.

2. Van Linschoten R, den Hoed PT, de Jongh AC. Guideline'Chronic Achilles tendinopathy, in particular tendinosis, in sportsmen/sportswomen'. Nederlands tijdschrift voor geneeskunde. 2007 Oct 20;151(42):2319.

3. Alfredson H. Chronic midportion Achilles tendinopathy: an update on research and treatment. Clinics in sports medicine. 2003 Oct 1;22(4):72741.

4. Kraushaar BS, Nirschl RP. Tendinosis of the elbow (tennis elbow): clinical features and findings of histological, immunohistochemical, and electron microscopy studies. Journal of Bone and Joint Surgery. 1999 Feb 1;81(2):259.

5. Kampa RJ, Connell DA. Treatment of tendinopathy: is there a role for autologous whole blood and platelet rich plasma injection? International journal of clinical practice. 2010 Dec;64(13):1813-23.

6. Monto RR. Platelet rich plasma treatment for chronic Achilles tendinosis. Foot \& Ankle International. 2012 May;33(5):379-85.

7. Paavola M, Kannus P, Paakkala T, Pasanen M, Järvinen M. Long-term prognosis of patients with Achilles tendinopathy. The American Journal of Sports Medicine. 2000 Sep;28(5):634-42.

8. Velnar T, Bailey T, Smrkolj V. The wound healing process: an overview of the cellular and molecular mechanisms. Journal of International Medical Research. 2009 Oct;37(5):1528-42.

9. Murphy G, Bretz U, Baggiolini M, Reynolds JJ. The latent collagenase and gelatinase of human polymorphonuclear neutrophil leucocytes. Biochemical Journal. 1980 Nov 15;192(2):517-25.

10. Eppley BL, Woodell JE, Higgins J. Platelet quantification and growth factor analysis from platelet-rich plasma: implications for wound healing. Plastic and reconstructive surgery. 2004 Nov 1;114(6):1502-8.

11. Maffulli N, Sharma P, Luscombe KL. Achilles tendinopathy: aetiology and management. Journal of the Royal Society of Medicine. 2004 Oct;97(10):472-6.

12. Josza LG, Kannus P. Human Kinetics Books; Champaign, IL, USA: 1997. Human tendons. anatomy, physiology, and pathology.:178-84.

13. Lian $\varnothing$, Refsnes PE, Engebretsen L, Bahr R. Performance characteristics of volleyball players with patellar tendinopathy. The American Journal of Sports Medicine. 2003 Mar;31(3):408-13.

14. Sconfienza LM, Silvestri E, Cimmino MA. Sonoelastography in the evaluation of painful achilles tendon in amateur athletes. Clin Exp Rheumatol. 2010; 28:373e8.

15. de Vos RJ, van Veldhoven PL, Moen MH, Weir A, Tol JL, Maffulli N. Autologous growth factor injections in chronic tendinopathy: a systematic review. Br Med Bull. 2010;95: 63e77.

16. Katsura T, Tohyama H, Kondo E, Kitamura N, Yasuda K. Effects of administration of trasforming growth factor (TGF)-betal and antiTGF-beta1 antibody on the mechanical properties of the stress-shielded patellar tendon. J Biomech. 2006; 39:2566e72.

17. Sheth U, Simunovic N, Klein G, Fu F, Einhorn TA, Schemitsch E, Ayeni OR, Bhandari M. Efficacy of autologous platelet-rich plasma use for orthopaedic indications: a meta-analysis. JBJS. 2012 Feb 15;94(4):298-307.

18. Magra M, Maffulli N. Nonsteroidal antiinflammatory drugs in tendinopathy: friend or foe. 2006

19. Andia I, Sanchez M, Maffulli N. Tendon healing and platelet-rich plasma therapies. Expert opinion on biological therapy. 2010 Oct 1;10(10):1415-26.

20. Abate M, Di Gregorio P, Schiavone C, Salini V, Tosi U. Platelet rich plasma in tendinopathies: how to explain the failure. Int $\mathbf{J}$ Immunopathol Pharmacol.2012; 25: 325-334.

21. Cole BJ, Seroyer ST, Filardo G, Bajaj S, Fortier LA. Platelet-rich plasma: where are we now and where are we going?. Sports health. 2010 May;2(3):203-10.

22. National Institute for Health and Clinical Excellence. Autologous Blood Injection for Tendinopathy: Guidance. http://www.nice.org.uk/nicemedia/pdf/IPG279G uidance. PDF (accessed November 2012)

23. De Vos RJ, Weir A, van Schie HT, BiermaZeinstra SM, Verhaar JA, Weinans H, Tol JL. Platelet-rich plasma injection for chronic Achilles tendinopathy: a randomized controlled trial. Jama. 2010 Jan 13;303(2):144-9.

24. Owens Jr RF, Ginnetti J, Conti SF, Latona C. Clinical and magnetic resonance imaging outcomes following platelet rich plasma injection for chronic midsubstance Achilles tendinopathy. Foot \& ankle international. 2011 Nov;32(11):1032-9. 